\title{
A CONJECTURE ON THE $p$-REGULARITY OF AN $\boldsymbol{H}$-SPACE
}

\author{
J. R. HUBBUCK
}

\begin{abstract}
It is shown that a compact, simply connected, Lie group $G$ is $p$-regular if and only if $K_{0}(\Omega G, Z / p Z)$ is a primitively generated polynomial algebra. It is conjectured that this remains true for $G$ a finite, simply connected, associative, $H$-space.
\end{abstract}

1. Let $G$ be a simply connected compact Lie group. There are well-known elegant theorems of Atiyah and Hirzebruch (Theorem 4.8 of [2]) and Hodgkin (Theorem $A$ of [7]) which describe the $Z / 2 Z$-graded complex $K^{*}$-theory ring structures $K^{*}(B G)$ and $K^{*}(G)$ and relate them to the representation theory of $G$. The first is a finitely generated power series ring naturally isomorphic to the completion of the character ring of $G$ with respect to the augmentation topology, the second is an exterior algebra on generators corresponding to the 'basic representations' of $G$. In contrast, and at first sight rather surprisingly, Clark has shown in [5] that $K^{*}(\Omega G)$ has no such simple description. However $K^{*}\left(\Omega G_{p}\right)$ is simpler and can reflect the homotopy of $G$ quite directly. ( $G_{p}$ is the localization of $G$ at the prime $p$ [6].) We illustrate this by considering Serre's concept of $p$-regularity. Recall that $G$ is defined to be $p$-regular if $G_{p}$ is homotopically equivalent to a produce of $p$-local spheres (Chapitre 5 of [14]). The Kumpel-Serre Theorem states that a simple $G$ is $p$-regular if and only if $p>(\operatorname{dim} G / \operatorname{rank} G)-1([14]$ and $[10])$.

THeOREM 1.1. Let $G$ be a simply connected, compact Lie group. Then $G$ is $p$-regular if and only if $K_{*}(\Omega G, Z / p Z)$ is a primitively generated polynomial algebra.

The 'only if' part of this result is clear and holds equally for ordinary homology, but the converse is false in the singular theory. The author has tried unsuccessfully to find a proof of this result which does not rely upon the classification of semisimple Lie groups. Now Lin has shown in Theorem 4.1.1 of [12] that the integral homology of the space of based loops on a simply connected finite $\boldsymbol{H}$-space has no odd torsion. This represents a major step forward in trying to establish analogues of the results of Atiyah, Hirzebruch and Hodgkin mentioned above when $G$ is replaced by a suitable finite $H$-space (see Theorem 4.6.3 of [12]). The conjecture of the title is that Theorem 1.1. remains true when $G$ is assumed only to be finite, associative

Received by the editors January 29, 1979.

AMS (MOS) subject classifications (1970). Primary 55D45; Secondary 55G25.

$K e y$ words and phrases. $p$-regularity of Lie groups, loop space, finite $\boldsymbol{H}$-space, $\boldsymbol{K}$-theory, Adams operator. 
$H$-space. This is true when $p$ is 2 and $H_{*}(\Omega G, Z)$ has no 2-torsion. (The associativity assumption in the conjecture cannot be removed.)

Let $X$ be a simply connected finite $H$-space. One can prove that $H_{*}(X, Z)$ has no $p$-torsion if and only if $H_{*}(\Omega X, Z / p Z)$ is a polynomial algebra on even dimensional generators and (assuming that $H_{*}(\Omega X, Z)$ has no 2-torsion if $p=2)$ this is equivalent to the requirement that $K_{*}\left(\Omega X, Z / p^{2} Z\right)$ is a polynomial algebra. Thus the proof of Theorem 1.1 is primarily concerned with the case when $H_{*}(X, Z)$ has no $p$-torsion. If we assume in addition that $H^{*}(X, Q)$ is a primitively generated exterior algebra, which will be the case if $X$ is homotopy associative, then a necessary (and sufficient) condition that $K_{*}(\Omega X, Z / p Z)$ is a primitively generated polynomial algebra is that the Adams operator $\psi^{p}: \tilde{K}^{*}\left(X, Z / p^{2} Z\right) \rightarrow \tilde{K}^{*}\left(X, Z / p^{2} Z\right)$ is zero. It is this condition which leads the author to the conjecture and which is used to establish it when $p=2$. Theorem 1.1 for Lie groups could be proved by this method but in the interests of brevity we do not follow it in detail.

Conditions sufficient to ensure that $X$ is $p$-regular have been given in [11]. Related work can be found in [15].

2. The notations used are standard, see [5] or [7]. We must show that if $G$ is not $p$-regular, then $K_{*}(\Omega G, Z / p Z)$ is not a primitively generated polynomial algebra. It is known that $H_{*}(\Omega G, Z)$ is torsion free and is zero in odd dimensions [3]. It has the structure of an associative, commutative, graded Hopf algebra. The corresponding properties therefore hold for $K_{*}(\Omega G, Z)$. Further $H^{*}(\Omega G, Z)$ and $H_{*}(\Omega G, Z)$ are the associative graded rings of $K^{*}(\Omega G, Z)$ and $K_{*}(\Omega G, Z)$ with the $C W$-filtrations.

Let $\xi_{K}: \tilde{K}^{*}(\Omega G, Z / p Z) \rightarrow \tilde{K}^{*}(\Omega G, Z / p Z)$ be the homomorphism defined by $\xi_{K}(u)=u^{p}$.

LEMMA 2.1. If $K_{*}(\Omega G, Z / p Z)$ is a primitively generated polynomial algebra, then $\xi_{K}=0$.

Proof. The properties of $H_{*}(\Omega G, Z)$ described above imply that

$$
K^{*}(\Omega G, Z / p Z) \cong \operatorname{Hom}\left\{K_{*}(\Omega G, Z / p Z), Z / p Z\right\}
$$

and the ring structure of $K^{*}(\Omega G, Z / p Z)$ is induced from the coalgebra structure of $K_{*}(\Omega G, Z / p Z)$. One can now prove the result by direct computation. Alternatively, $K_{*}(\Omega G, Z / p Z) \cong K_{*}(\Omega Y, Z / p Z)$ as Hopf algebras, where $Y$ is a finite produce of odd dimensional spheres. But $\xi_{K}$ : $\tilde{K}^{*}(\Omega Y, Z / p Z) \rightarrow \tilde{K}^{*}(\Omega Y, Z / p Z)$ is zero, since $K^{*}(\Omega Y, Z)$ is a divided power series ring.

Let $\xi_{H}: \tilde{H}^{*}(\Omega G, Z / p Z) \rightarrow \tilde{H}^{*}(\Omega G, Z / p Z)$ be defined by $\xi_{H}(x)=x^{p}$.

COROLlaRY 2.2. Under the hypotheses of Lemma 2.1, $\xi_{H}=0$.

LEMMA 2.3. Suppose that $P^{1}: H^{3}(G, Z / p Z) \rightarrow H^{2 p+1}(G, Z / p Z)$ is nonzero. Then $\xi_{H} \neq 0$. (If $p=2$, interpret $P^{1}$ as $\mathrm{Sq}^{2}$.) 
Proof. Here and below, we appeal to Theorem 5.14 of [4]. There is a commutative diagram

$$
\begin{aligned}
H^{3}(G, Z / p Z) \cong P H^{3}(G, Z / p Z) \cong & Q H^{3}(G, Z / p Z) \stackrel{P^{1}}{\rightarrow} Q H^{2 p+1}(G, Z / p Z) \\
\downarrow \sigma^{*} & \sigma^{*} \\
& P H^{2}(\Omega G, Z / p Z) \stackrel{P^{1}}{\rightarrow} P H^{2 p}(\Omega G, Z / p Z)
\end{aligned}
$$

and the cohomology suspensions are isomorphisms in these dimensions. The hypothesis implies that $P^{1}$ on the indecomposable quotient module is nonzero, for $P^{1}$ of a primitive element is primitive and a nonzero primitive of dimension $2 p+1$ is indecomposable. Thus $P^{1}$ in the bottom line is nonzero and in dimension two, $P^{1}$ coincides with $\xi_{H}$.

The decomposition theorem for simply connected, semisimple Lie groups implies that $G$ is a product of copies of $\operatorname{SU}(n), \operatorname{Sp}(n)$ and $\operatorname{Spin}(n)$ for different values of $n$ and $G_{2}, F_{4}, E_{6}, E_{7}$ and $E_{8}$. If we exclude the cases when $p=2$ and $G$ is $\bmod 2$ equivalent to a product of $\operatorname{Sp}(n)$ 's, $n>1$, and spheres, and when $p=3$ and $G$ is $\bmod 3$ equivalent to a product of $G_{2}$ 's and spheres, the proof of Theorem 1.1 follows easily. It is sufficient to consider a simple $G$. But if $G$ is not $p$-regular, $P^{1}: H^{3}(G, Z / p Z) \rightarrow H^{2 p+1}(G, Z / p Z)$ is not zero and the result follows from Corollary 2.2 and Lemma 2.3. Convenient references for the information on $P^{1}$ (except for the fact that $\mathrm{Sq}^{2}: H^{3}(\mathrm{SU}(n), Z / 2 Z) \rightarrow$ $H^{5}(\mathrm{SU}(n), Z / 2 Z), n>2$, is nonzero) are Theorem 1.1 of [13] if $H_{*}(G, Z)$ has no $p$-torsion and III, §2, Proposition 2.2 and (3.1) of [7] when $H_{*}(G, Z)$ has $p$-torsion.

3. The Kunneth formula for $K_{*}(\Omega G, Z / p Z)$ implies that to complete the proof of Theorem 1.1, it is sufficient to show that neither $K_{*}\left(\Omega G_{2}, Z / 3 Z\right)$ nor $K_{*}(\Omega \mathrm{Sp}(n), Z / 2 Z)$, for $n>1$, is a primitively generated polynomial algebra. This information can be extracted from [5] but we shall give an $H$-space proof.

In all cases $H_{*}(G, Z)$ has no $p$-torsion and so by Theorem 5.14 of [4],

$$
\sigma^{*}: Q H^{*}(G, Z / p Z) \rightarrow P H^{*}(\Omega G, Z / p Z)
$$

is an isomorphism. It follows that

$$
\sigma^{*}: Q K^{*}(G, Z / p Z) \rightarrow P K^{*}(\Omega G, Z / p Z)
$$

is an isomorphism. This is a routine verification with the spectral sequence of [2], using the fact that there is a commutative square

$$
\begin{array}{ccc}
\tilde{H}^{* *}(G, Q) & \stackrel{\sigma^{*}}{\rightarrow} & \tilde{H}^{* *}(\Omega G, Q) \\
\uparrow \mathrm{ch} & & \uparrow \mathrm{ch} \\
\tilde{K}^{*}\left(G, Q_{p}\right) & \stackrel{\sigma^{*}}{\rightarrow} & \tilde{K}^{*}\left(\Omega G, Q_{p}\right)
\end{array}
$$

where $Q_{p}$ is the subring of rational numbers with denominators prime to $p$. Now consider the Adams operator $\psi^{p}: \tilde{K}^{*}\left(G, Q_{p}\right) \rightarrow \tilde{K}^{*}\left(G, Q_{p}\right)$ and recall that $\psi^{p}(u)=u^{p} \bmod p$. Since $K^{*}\left(G, Q_{p}\right)$ is an exterior algebra over $Q_{p}$, there 
is a well-defined linear map

$$
p^{-1} \psi^{p}: \tilde{K}^{-1}(G, Z / p Z) \rightarrow \tilde{K}^{-1}(G, Z / p Z) .
$$

The definition of $\sigma^{*}$ implies that the following square is commutative:

$$
\begin{array}{ccc}
\tilde{K}^{-1}(G, Z / p Z) & \stackrel{p^{-1} \psi^{p}}{\rightarrow} & \tilde{K}^{-1}(G, Z / p Z) \\
\downarrow \sigma^{*} & & \downarrow \sigma^{*} \\
\tilde{K}^{0}(\Omega G, Z / p Z) & \stackrel{\psi^{p}}{\rightarrow} & \tilde{K}^{0}(\Omega G, Z / p Z)
\end{array}
$$

But $\psi^{p}=\xi_{K}$ in the bottom line and so by Lemma 2.1 and (3.1), to establish that $K_{*}(\Omega G, Z / p Z)$ is not a primitive polynomial algebra, it is sufficient to check that $p^{-1} \psi^{p}: \tilde{K}^{-1}(G, Z / p Z) \rightarrow \tilde{K}^{-1}(G, Z / p Z)$ is nonzero, or equivalently that $\psi^{p}: \tilde{K}^{*}\left(G, Q_{p}\right) \rightarrow \tilde{K}^{*}\left(G, Q_{p}\right)$ is nonzero $\bmod p^{2}$.

Consider first $p=3$ and $G=G_{2}$. We assume only that $G$ is an associative $H$-space and that $H^{*}(G, Z / 3 Z) \cong E\left(x_{3}, x_{11}\right)$, an exterior algebra over $Z / 3 Z$ on generators of dimensions 3 and 11. (However, this is not a significant generalization. The spaces of rank 2 simply connected finite $H$-spaces have been classified and our hypotheses imply that any such $G$ is $\bmod 3$ equivalent to $G_{2}$.) We shall use the language of [8].

It is well known that $H^{*}\left(B G, Q_{3}\right) \cong Q_{3}\left[y_{4}, y_{12}\right]$ and $K^{*}\left(B G, Q_{3}\right) \cong$ $Q_{3}\left[\left[u_{4}, u_{12}\right]\right]$, a power series ring on generators of exact $C W$-filtrations 4 and 12 which are representatives for $y_{4}$ and $y_{12}$ in the associated graded ring. Let $J: H^{\text {even }}\left(B G, Q_{3}\right) \rightarrow K^{0}\left(B G, Q_{3}\right)$ be a ring isomorphism defined by setting $J\left(x_{4}\right)=u_{4}$ and $J\left(x_{12}\right)=u_{12}$. There are homomorphisms $Q_{J}^{q}, S_{J}^{q}: H^{2 n}\left(B G, Q_{3}\right)$ $\rightarrow H^{2 n+2 q}\left(B G, Q_{3}\right)$ defined by setting $Q_{J}^{q} x=2^{q} c h_{n+q} J(x)$ and $\Sigma S_{J}^{i} Q_{J}^{q-i}=0$ $(0<i<q)$ where $S_{J}^{0}$ is the identity. We now apply Corollary 2.12 and Lemma 2.14 of [8]. In the notation introduced just before these results

$$
\left(1-k^{12}\right) S_{J}^{6} y_{12}=k^{8} T^{2}(k) S_{J}^{4} y_{12}+k^{10} T^{1}(k) S_{J}^{5} y_{12} \bmod 27
$$

where 3 does not divide $k$. We pass to the quotient ring $H^{\text {even }}\left(B G, Q_{3}\right) /\left\{\bar{H}^{\text {even }}\left(B G, Q_{3}\right)\right\}^{4}$ and set $k=2$. Using the fact that $S_{J}^{6} y_{12}=$ $y_{12}^{3} \bmod 3$, for dimensional reasons it follows that

$$
9 y_{12}^{3}=21 S_{J}^{2} S_{J}^{4} y_{12} \bmod 27 .
$$

But $S_{J}^{4} y_{12}=\alpha y_{4} y_{12}^{2}$ for some $\alpha \in Q_{3}$. The cartan formula for $S_{J}^{q}$ implies that $S_{J}^{2} y_{4}=3 y_{12} \bmod 9$ in $Q H^{\text {even }}\left(B G, Q_{3}\right)$ and directly from the definitions we deduce that $\psi^{3}\left(u_{4}\right)=3 u_{12} \bmod 9$ in $Q K^{0}\left(B G, Q_{3}\right)$. The inclusion $S G \rightarrow B G$ induces an isomorphism $Q K^{0}\left(B G, Q_{3}\right) \rightarrow K^{-1}\left(G, Q_{3}\right)$ and therefore $\psi^{3}$ : $K^{*}\left(G, Q_{3}\right) \rightarrow K^{*}\left(G, Q_{3}\right)$ is not zero $\bmod 9$.

There is a similar argument which deals with $\operatorname{Sp}(n), n>1$ and $p=2$. This was hinted at in [9], although what we require here is much simpler, and so we shall not give the details. Alternatively, the 8 skeleton of $\operatorname{Sp}(n), n>1$ is $S^{3} \cup e^{7}$ where the 7-cell is attached by $\omega$, a generator of $\pi_{6}\left(S^{3}\right) \simeq Z_{12}$. Following [1], but working unstably, one can consider the exact sequence

$$
0 \rightarrow \tilde{K}^{-1}\left(S^{7}\right) \rightarrow \tilde{K}^{-1}\left(S^{3} \cup e^{7}\right) \rightarrow \tilde{K}^{-1}\left(S^{3}\right) \rightarrow 0 .
$$


Since $e_{C}(\omega)=6^{-1}$, it follows as in $\$ 7$ of [1] that $\psi^{2}(u)=2 v \bmod 4$ in $\tilde{K}^{-1}\left(S^{3} \cup e^{7}\right)$ where $u$ maps to a generator of $\tilde{K}^{-1}\left(S^{3}\right)$ and $v$ is the image of a generator of $\tilde{K}^{-1}\left(S^{7}\right)$. This is sufficient to imply that $\psi^{2}: K^{-1}(\operatorname{Sp}(n)) \rightarrow$ $K^{-1}(\mathrm{Sp}(n))$ is nonzero $\bmod 4$.

\section{REFERENCES}

1. J. F. Adams, On the groups $J(X)$. IV, Topology 5 (1966), 21-71.

2. M. F. Atiyah and F. Hirzebruch, Vector bundles and homogeneous spaces, Proc. Sympos. Pure Math., vol. 3, Amer. Math. Soc., Providence, R. I., 1961, pp. 7-38.

3. R. Bott, On torsion in Lie groups, Proc. Nat. Acad. Sci. U.S.A. 40 (1954), 586-588.

4. W. Browder, On differential Hopf algebras, Trans. Amer. Math. Soc. 107 (1963), 153-176.

5. Francis Clark, On the K-theory of the loop space of a Lie group, Proc. Cambridge Philos. Soc. 76 (1974), 1-20.

6. P. Hilton, G. Mislin and J. Roitberg, Localization of nilpotent groups and spaces, Notas de Mathematica 15, North-Holland Math. Studies, North-Holland, Amsterdam, 1975.

7. Luke Hogkin, On the K-theory of Lie groups, Topology 6 (1967), 1-36.

8. J. R. Hubbuck, Primitivity in torsion free cohomology Hopf algebras, Comment. Math. Helv. 46 (1971), 13-43.

9. , Two examples of finite $H$-spaces, Geometric Applications of Homotopy Theory 1 , Lecture Notes in Math., vol. 657, Springer-Verlag, Berlin and New York, 1978, pp. 282-291.

10. P. G. Kumpel, Jr., Lie groups and products of spheres, Proc. Amer. Math. Soc. 16 (1965), $1350-1356$.

11. On p-equivalences of mod $p H$-spaces, Quart. J. Math. Oxford Ser. (2) 23 (1972), 173-178.

12. J. Lin, Torsion in H-spaces. II, Ann. of Math. (2) 107 (1978), 41-88.

13. M. Mimura and H. Toda, Cohomology operations and the homotopy of compact Lie groups, Topology 9 (1970), 317-336.

14. J. P. Serre, Groupes d'homotopie et classes de groupes abéliens, Ann. of Math. (2) 58 (1953), 248-294.

15. C. Wilkerson, $K$-theory operations and mod p loop spaces, Math. Z. 132 (1973), 29-44.

Department of Mathematics, University of aberdeen, Edward Wright Building, ABERDEEN, SCOTLAND 